\title{
Constitutional mismatch repair deficiency in childhood colorectal cancer harboring a de novo variant in the MSH6 gene: a case report
}

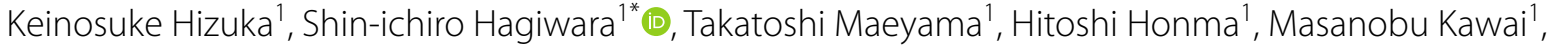
Kiwamu Akagi ${ }^{2}$, Michiko Yasuhara ${ }^{3}$, Naohiro Tomita ${ }^{3,4}$ and Yuri Etani ${ }^{1}$

\begin{abstract}
Background: Constitutional mismatch repair deficiency (CMMRD) is caused by biallelic pathogenic variants in one of the mismatch repair genes, and results in early onset colorectal cancer, leukemia, brain tumors and other childhood malignancies. Here we report a case of CMMRD with compound heterozygous variants in the MSH6 gene, including a de novo variant in multiple colorectal cancers.

Case presentation: An 11-year-old girl, who presented with multiple spots resembling café-au-lait macules since birth, developed abdominal pain, diarrhea and bloody stool over two months. Colonoscopy revealed multiple colonic polyps, including a large epithelial tumor, and pathological examination revealed tubular adenocarcinoma. Brain magnetic resonance imaging (MRI) showed an unidentified bright object (UBO), commonly seen in neurofibromatosis type 1 (NF1). Genetic testing revealed compound heterozygous variants, c. [2969T > A (p.Leu990*)] and [3064G > T (p.Glu1022*)] in the MSH6 gene; c.2969T > A (p.Leu990*) was identified as a de novo variant.
\end{abstract}

Conclusions: We present the first report of a CMMRD patient with a de novo variant in MSH6, who developed colorectal cancer in childhood. CMMRD symptoms often resemble NF1, as observed here. Physicians should become familiar with CMMRD clinical phenotypes for the screening and early detection of cancer.

Keywords: Café-au-lait macules, Case report, Constitutional mismatch repair deficiency, Mismatch repair gene, Neurofibromatosis type 1

\section{Background}

Constitutional mismatch repair deficiency (CMMRD; Online Mendelian Inheritance in Man; \#276300) is a childhood cancer predisposition syndrome caused by biallelic pathogenic variants in one of four mismatch repair (MMR) genes, i.e., $M L H 1, M S H 2, M S H 6$ and PMS2 [1]. MMR genes recognize and repair mismatched nucleotides, insertions, and deletions during DNA

\footnotetext{
*Correspondence: hagi114@wch.opho.jp

${ }^{1}$ Department of Gastroenterology, Nutrition and Endocrinology,

Osaka Women's and Children's Hospital, 840 Murodo-cho, Izumi, Osaka

594-1101, Japan

Full list of author information is available at the end of the article
}

replication. MMR deficiency facilitates the accumulation of genetic changes and consequently, cancer development [2]. From the literature, few case reports have outlined de novo variants in CMMRD. Here, we report a patient with CMMRD who developed multiple colorectal cancers in childhood. The cancer was characterized by compound heterozygous variants in MSH6, including a de novo variant.

\section{Case presentation}

An 11-year-old girl exhibited persistent abdominal pain, diarrhea and bloody stool for two months. A bacterial infection was suspected and she was treated with antibiotics, but her symptoms persisted and she 
was referred to our hospital. Up to two years old, her medical history indicated she had undergone repeated ophthalmological examinations and brain magnetic resonance imaging (MRI) for suspected neurofibromatosis type 1 (NF1) because of multiple café-au-lait macules (CALM) present since birth. A family history showed her maternal grandmother had breast cancer in her $50 \mathrm{~s}$, but no other history of CALM and/or colorectal cancer was recorded (Fig. 1). At admission, a physical examination revealed CALM on the dorsal area and the lower limbs (Fig. 2a, b). She had lost $3 \mathrm{~kg}$ in two months. Laboratory tests revealed mild anemia, and other blood tests were within normal limits. An ultrasonography demonstrated a mass protruding into the lumen of the descending colon. Colonoscopy (CS) revealed multiple polyps in the sigmoid and descending colon. The colonoscope could not pass the descending colon because the epithelial tumor occupied most of the colon lumen. Histology of the polyps revealed tubular adenoma (Group 3). Subsequent contrast-enhanced computed tomography showed a mass lesion in the ascending colon. A brain MRI showed faint T2-hyperintensities in the left cerebellar hemisphere, right pons, and right globus pallidus,
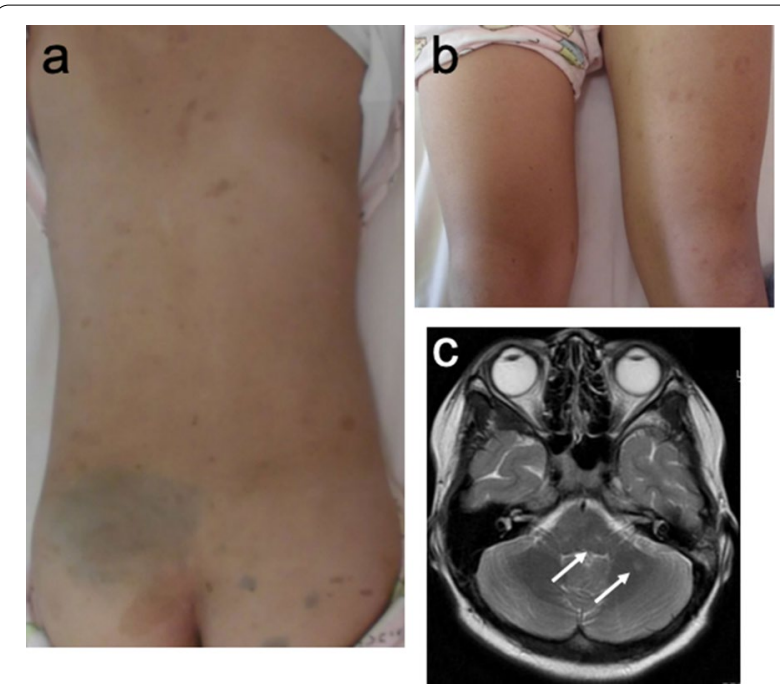

Fig. 2 Café-au-lait macules (CALM) with irregular boundaries and uneven pigmentation on the dorsal area (a) and lower limbs (b). c Magnetic resonance imaging (MRI); arrows indicate unidentified bright objects (UBO) in the left cerebellar hemisphere

indicating an unidentified bright object (UBO), potentially indicative of NF1 (Fig. 2c). No neoplastic lesions were observed.

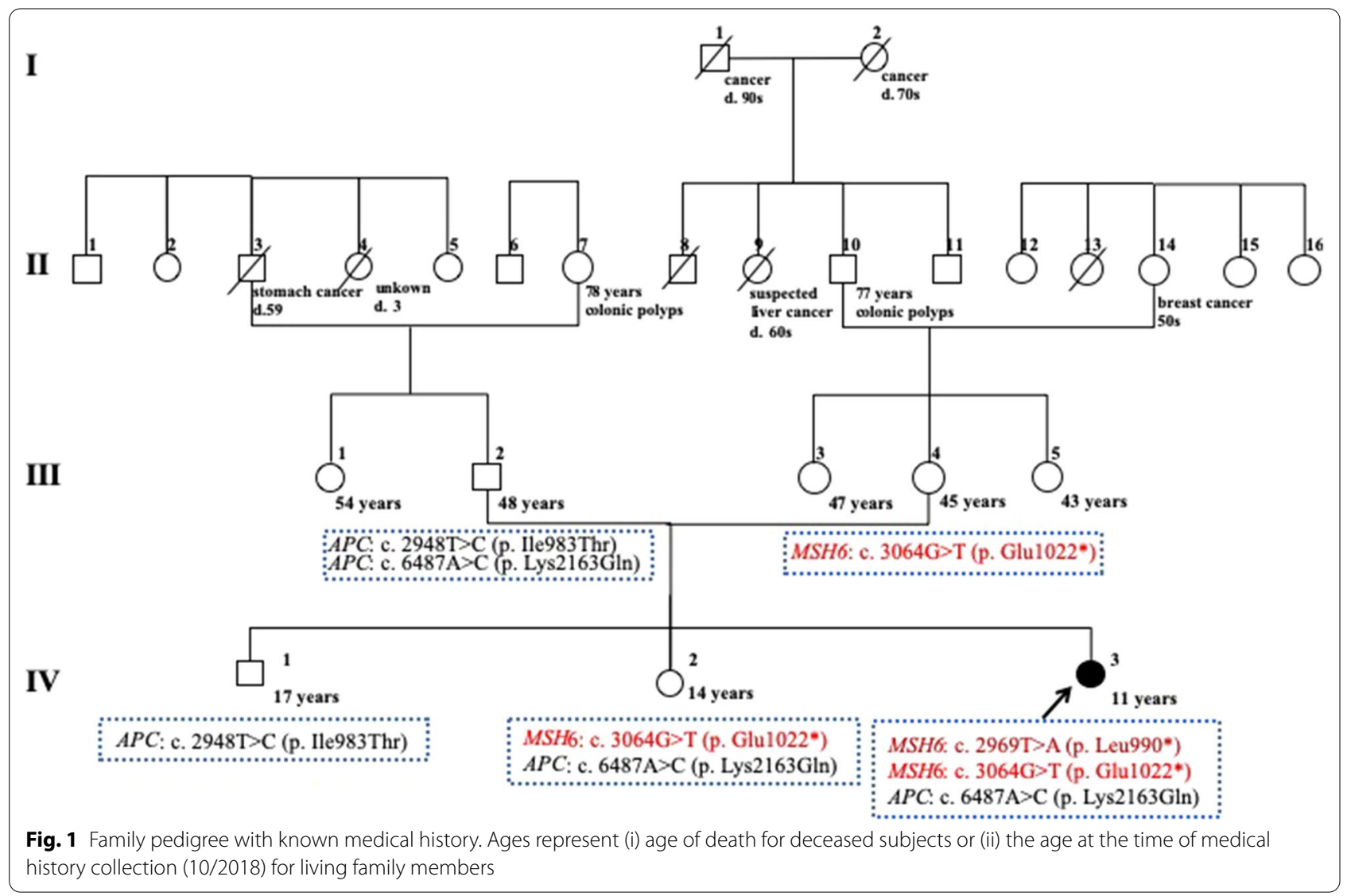


Based on these endoscopic findings, it was highly likely the tumor in the descending colon was colorectal cancer. Approximately three weeks after admission, she was transferred to a university hospital to treat the colon polyps. After transfer, a second CS was successful for cecal intubation. This revealed multiple polyps in the transverse colon and ascending colon, and biopsy sampling revealed adenocarcinoma (tub1/tub2). One week later, polyps were removed by endoscopic mucosal resection and submucosal dissection. Pathological examination of four of the 19 polyps revealed moderately well differentiated tubular adenocarcinoma. Surgery was scheduled to remove the large tumor of the descending colon at a later date because it could not be removed endoscopically. Prior to surgery, the patient developed abdominal distension. MRI showed ascites and bilateral ovarian cysts with solid materials. Ascitic drainage was performed.

Approximately four weeks after transfer, the patient underwent laparoscopic surgery. Bilateral ovariectomy was performed in addition to left hemicolectomy as adenocarcinomas were identified in ovarian solid materials, even though ascitic fluid cytology was negative for malignant cells (Fig. 3a, b).

Pathology of the resected colon and bilateral ovaries revealed adenocarcinoma. Histologically, it was difficult to determine whether the ovarian tumors were concurrent or metastatic from the primary tumor. The tumor of the descending colon had invaded from the muscularis propria to pericolorectal tissues. Lymph node metastasis was observed in one location (pT3N1aM1a, pStage IV).

We also performed microsatellite instability (MSI) testing of genomic DNA from micro-dissected samples of the colorectal adenocarcinoma. This was conducted using the first panel of five microsatellite markers as recommended by the National Cancer Institute Consensus Conference [3]. The colorectal adenocarcinoma was classified as MSI-high (4/5). Immunohistochemical (IHC) tumor analysis indicated absent MSH2 and MSH6 protein expression, although MLH1 and PMS2 expression was positive. Taken together, CMMRD or Lynch syndrome (LS) was suspected. To be diagnostically definitive, we performed a multi-gene panel assay which contained 14 genes involved in hereditary colorectal cancer syndromes; MLH1, MSH2, MSH6, PMS2, EPCAM, MSH3, MLH3, APC, MUTYH, POLD1, POLE, TP53, $M B D 4$, and NTHL1. The results revealed biallelic variants in MSH6; c. [2969 T > A (p.Leu990*)] and [3064G > T (p.Glu1022*)] (Figs. 1, 4). From this genetic information, the patient was diagnosed with CMMRD, indicating compound heterozygous variants in MSH6. This result suggested that loss of $\mathrm{MSH} 2$ protein expression in tumor tissue was probably due to secondary acquired somatic changes in $M S H 2$. Parental and sibling genetic testing confirmed the patient was the biological daughter of her parents and an offspring of a non-consanguineous marriage. Her mother and sister had one variant of
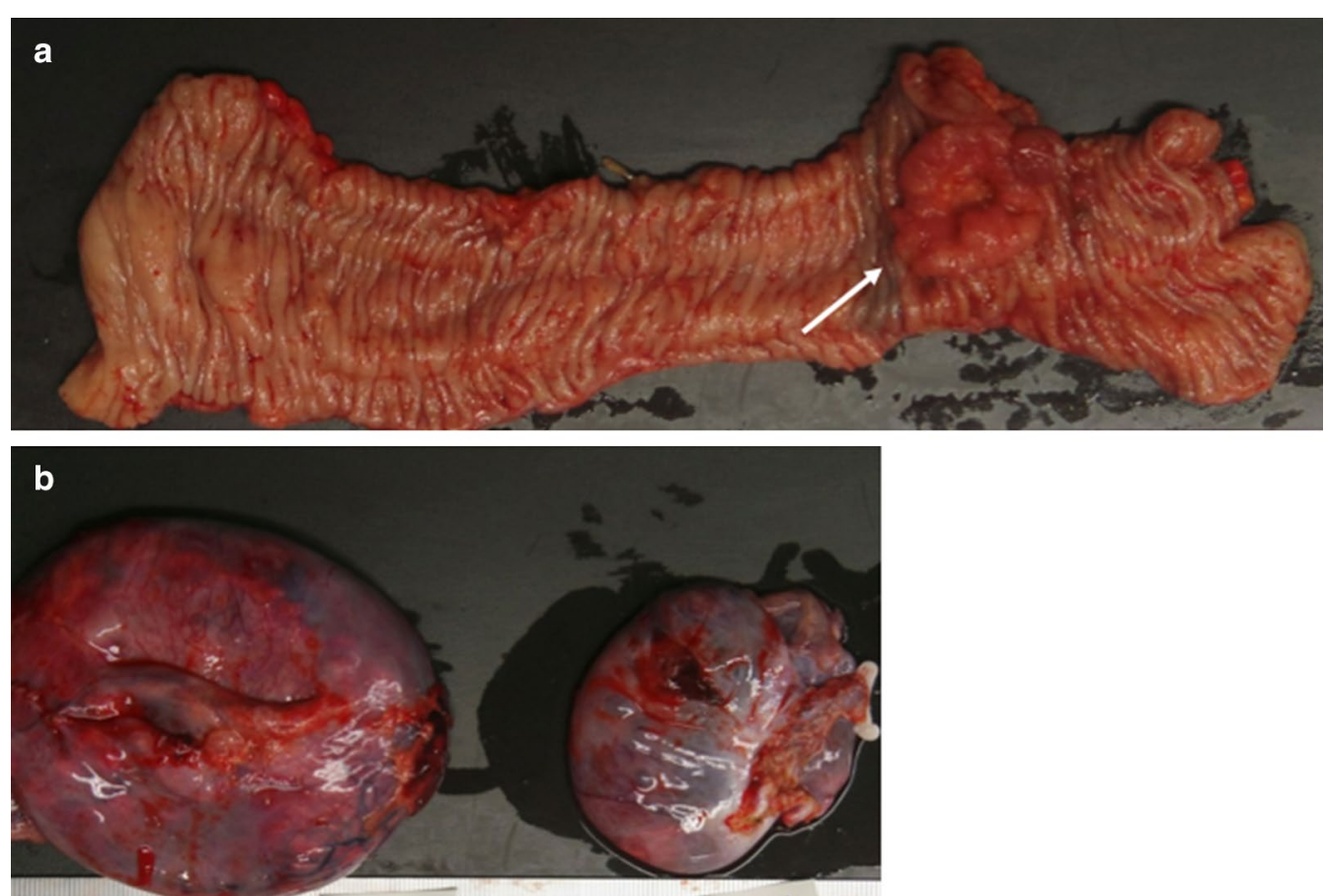

Fig. 3 Macroscopic findings. a The arrow indicates the tumor in the descending colon. b The bilateral ovarian cysts with solid materials 


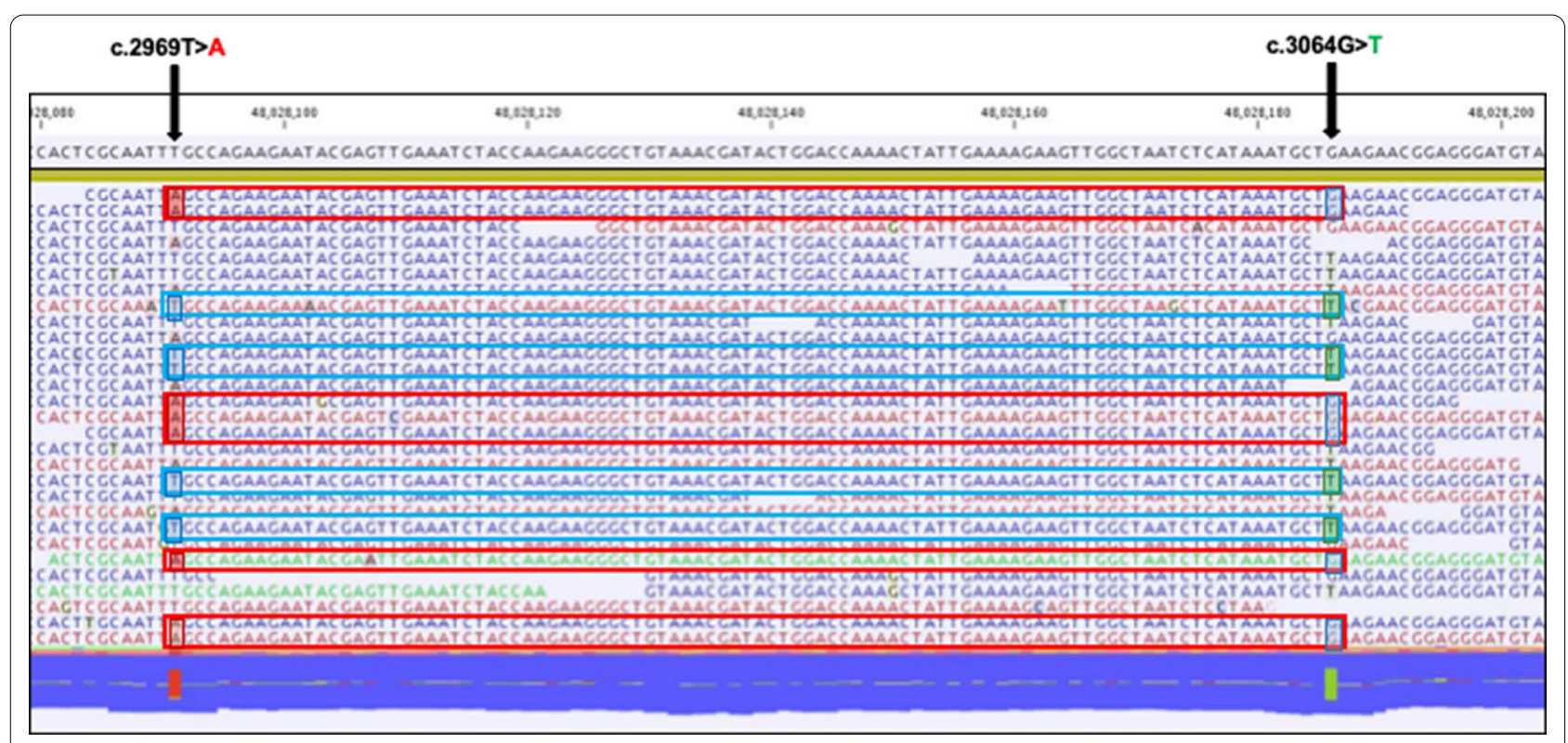

Fig. 4 Biallelic variants in MSH6 were identified using multi-gene analysis. Genetic testing revealed compound heterozygous variants; C. [2969T > A (p.Leu990*)] and [3064G > T (p.Glu1022*)] in MSH6

MSH6, c.3064G > T (p.Glu1022*), but the other variant, c.2969 T > A (p.Leu990*) was not identified in her parents, brother and sister. Her mother and sister who had the c.3064G $>\mathrm{T}$ variant never had LS-related tumors. These genetic analyses indicated the second variant, c.2969 T > A (p.Leu990*) had occurred de novo.

\section{Discussion and conclusions}

We reported the case of CMMRD caused by two pathogenic variants in MSH6, one of which was de novo.

Childhood colorectal cancer (CRC) is extremely rare, with an incidence of approximately 1-3 cases per million in the United States [4]. Up to 2003, Yasuoka et al. reported a total of 94 childhood CRC cases in Japan [5]. The pathogenic mechanism of childhood CRC is distinct to adults because it develops independent of lifestyle, with a very poor prognosis. Studies have reported that $10 \%-30 \%$ of childhood CRC cases are related to predisposing factors $[6,7]$. These include familial adenomatous polyposis, ulcerative colitis, Crohn's disease, and Peutz-Jeghers syndrome [6]. Furthermore, limited case reports have shown that childhood CRC and adolescent LS, which is a hereditary cancer syndrome, are caused by a monoallelic pathogenic variant in one of the MMR genes [8-10]. Our case was suspected with a hereditary predisposition to cancer because she developed CRC at 11 years old. For this reason, she underwent genetic testing and was diagnosed with CMMRD.

CMMRD is a childhood cancer predisposition syndrome caused by biallelic homozygous or compound heterozygous pathogenic variants in one of the MMR genes [1]. The age at diagnosis of an initial CMMRD malignancy ranges between 0.4 and 39 years of age, with approximately $80 \%$ of cases occurring under 18 years old [11]. The tumor spectrum for CMMRD mainly includes hematological malignancies, brain/central nervous system tumors, LS-associated tumors, and other malignancies [2]. LS-associated tumors include colorectal, small bowel, endometrial, ureter, renal pelvis, biliary tract, stomach and bladder carcinoma [11]. The age of onset and tumor spectrum varies depending on the MMR genes [11].

Our case showed compound heterozygous pathogenic variants in MSH6; c.[2969 T>A (p.Leu990*)] and $[3064 \mathrm{G}>\mathrm{T}$ (p.Glu1022*)]. The c.3064G $>\mathrm{T}$ variant was present in her mother and sister. However, the c.2969 T > A variant was identified in neither parent. The patient also had a variant in $A P C$, c. $[6487 \mathrm{~A}>\mathrm{C}$ (p.Lys2163Gln)]. The allele frequency of this variant is 0.00002 according to the Genome Aggregation Database [12]. Her father also had the same rare APC variant, indicating she was her parent's biological daughter.

Taken together, c.2969T $>$ A was considered a de novo variant. Both c.3064G > T and c.2969T > A variants have previously been identified as pathogenic variants [13]. To the best of our knowledge, this is the first report indicating that one of the alleles in MSH6 was a de novo variant in a pediatric CRC case, although a CMMRD patient with a de novo variant of the same gene, who developed B-cell acute lymphoblastic leukemia was previously reported 
[14]. Win et al. reported six de novo variant cases (in MSH6) in 261 LS patients [15]. Our investigation suggests that de novo variants in MMR genes are extremely rare in CMMRD.

CMMRD shows a wide range of clinical manifestations [11]. Patients display clinical features reminiscent of NF1 [16]. Multiple CALM was reported in $62 \%$ of CMMRD patients, and approximately $30 \%$ had other NF1 features (i.e., freckling, neurofibroma, lisch nodules, and UBO) [8]. It has been hypothesized that NF1 is a frequent somatic target for MMR deficiency due to biallelic pathogenic variants in one of the MMR genes [17]. In support of this theory, studies have shown somatic NF1 variants in MSI-high cell lines and MSI-high primary CRCs [17]. However, CALM in most CMMRD cases is $<$ six occurrences; CALM in CMMRD exhibit irregular boundaries and uneven pigmentation, unlike the typical CALM in NF1 [8]. A recent study suggested that CALM in CMMRD could be distinguished from CALM in NF1 by experienced physicians, however some CMMRD cases exhibited typical NF1 CALM [16]. Furthermore, if an individual had atypical CALM, other CALM associated diseases (e.g., Legius syndrome, Noonan syndrome, and McCune-Albright syndrome) should be considered [18]. In our case, the patient showed UBO and atypical CALM. Since birth, she had undergone extensive tests for suspected NF1. She should have been screened for CMMRD because her CALM exhibited atypical features of NF1, e.g., irregular boundaries and uneven pigmentation. Suerink et al. reported that a 6-year-old girl without tumors was diagnosed with CMMRD [1]. Even though she met the diagnostic criteria for NF1, she did not exhibit NF1 variants. Thus, she was suspected of having CMMRD, and genetic testing showed biallelic pathogenic variants in PMS2. In cases with NF1-like symptoms without NF1 variants or atypical CALM, CMMRD must be considered [19].

As CMMRD was first reported in 1999 [20, 21], further cases must be identified and investigated. Cases of suspicious NF1 or early childhood death from hematological malignancies or brain tumors may be undiagnosed for CMMRD. Going forward, it is important to inform physicians of CMMRD to allow patients receive appropriate clinical treatment and surveillance.

\section{Abbreviations \\ CMMRD: Constitutional mismatch repair deficiency; MMR: Mismatch repair; NF1: Neurofibromatosis type 1; CALM: Café-au-lait macules; CS: Colonoscopy; UBO: Unidentified bright object; MSI: Microsatellite instability; IHC: Immuno- histochemical; CRC: Colorectal cancer; LS: Lynch syndrome.}

\section{Acknowledgements}

The authors are very grateful to Prof Y. Nakayama (Shinshu University School of Medicine) and Dr H. Ishikawa (Kyoto Prefectural University of Medicine) for advice on diagnosis and treatment.

\section{Authors' contributions}

$\mathrm{KH}, \mathrm{SH}, \mathrm{KA}$, and NT contributed to manuscript drafting. $\mathrm{KH}, \mathrm{SH}, \mathrm{KA}$, and NT were responsible for data collection. $\mathrm{KH}, \mathrm{SH}, \mathrm{TM}, \mathrm{HH}, \mathrm{MK}, \mathrm{MY}, \mathrm{NT}$, and YE participated in patient management. All authors read and approved the final manuscript.

\section{Funding}

This research was supported by the Dial Study from the Japan Agency for Medical Research and Development, AMED. The research was also supported by Japan AMED under the grant reference; JP $18 \mathrm{kk0205004}$ and JSPS KAKENHI grant reference; JP18K07339.

\section{Availability of data and materials}

Not applicable.

\section{Ethics approval and consent to participate}

Not applicable.

\section{Consent for publication}

Written informed consent was obtained from the patient's parents for publication. The consent document is available for editorial review.

\section{Competing interests}

None of the authors declare any conflict of interest.

\section{Author details}

${ }^{1}$ Department of Gastroenterology, Nutrition and Endocrinology, Osaka Women's and Children's Hospital, 840 Murodo-cho, Izumi, Osaka 594-1101, Japan. ${ }^{2}$ Division of Molecular Diagnosis and Cancer Prevention, Saitama Cancer Center, 780, Komuro, Ina-machi, Kitaadachi-gun, Saitama 780362-0806, Japan. ${ }^{3}$ Division of Lower GI Surgery, Department of Surgery, Hyogo College of Medicine, 1-1 Mukogawa-cho, Nishinomiya, Hyogo 663-8501, Japan. ${ }^{4}$ Cancer Treatment Center, Toyonaka Municipal Hospital, 4-14-1, Shibahara-cho, Toyonaka, Osaka 560-8565, Japan.

Received: 5 November 2020 Accepted: 4 February 2021

Published online: 10 February 2021

\section{References}

1. Suerink M, Potjer TP, Versluijs AB, Ten Broeke SW, Tops CM, Wimmer K, Nielsen $M$, et al. Constitutional mismatch repair deficiency in a healthy child: on the spot diagnosis? Clin Genet. 2018:93:134-7.

2. Wimmer K, Etzler J. Constitutional mismatch repair-deficiency syndrome: have we so far seen only the tip of an iceberg? Hum Genet. 2008;124:105-22.

3. Boland CR, Thibodeau SN, Hamilton SR, Sidransky D, Eshleman JR, Burt RW, et al. A National Cancer Institute Workshop on Microsatellite Instability for cancer detection and familial predisposition: development of international criteria for the determination of microsatellite instability in colorectal cancer. Cancer Res. 1998;58:5248-57.

4. SEER*Explorer: An interactive website for SEER cancer statistics [Internet]. Surveillance Research Program, National Cancer Institute. 2020. https:// seer.cancer.gov/explorer/. Accessed 8 August 2020.

5. Yasuoka R, Kumano T, Morita S, Mitsuo M, Oda T, Kawabata K, et al. A case of colon cancer in a 15-year-old boy. J Jpn Surg Assoc. 2005;66:129-34.

6. Karnak I, Ciftci AO, Senocak ME, Buyukpamukcu N. Colorectal carcinoma in children. J Pediatr Surg. 1999;34:1499-504.

7. Salas-Valverde S, Lizano A, Gamboa Y, Vega S, Barrantes M, Santamaría S, Zamora JB. Colon carcinoma in children and adolescents: prognostic factors and outcome-a review of 11 cases. Pediatr Surg Int. 2009;25:1073-6.

8. Niessen RC, Berends MJ, Wu Y, Sijmons RH, Hollema H, Ligtenberg MJL, et al. Identification of mismatch repair gene mutations in young patients with colorectal cancer and in patients with multiple tumours associated with hereditary non-polyposis colorectal cancer. Gut. 2006;55:1781-8.

9. Bodas A, Perez-Segura P, Maluenda C, Caldes T, Olivera E, Diaz-Rubio E. Lynch syndrome in a 15-year-old boy. Eur J Pediatr. 2008;167:1213-5. 
10. Huang SC, Lavine JE, Boland PS, Newbury RO, Kolodner R, Pham TT, et al. Germline characterization of early-aged onset of hereditary non-polyposis colorectal cancer. J Pediatr. 2001;138:629-35.

11. Wimmer K, Kratz CP, Vasen HF, Caron O, Colas C, Entz-Werle N, et al. Diagnostic criteria for constitutional mismatch repair deficiency syndrome: suggestions of the European consortium "care for CMMRD" (C4CMMRD). J Med Genet. 2014;51:355-65.

12. Genome Aggregation Database. https://gnomad.broadinstitute.org/. Accessed 30 Sep 2020.

13. Clin Var. https://www.ncbi.nlm.nih.gov/clinvar/. Accessed 24 Dec 2020.

14. Tesch VK, IJspeert H, Raicht A, Rueda D, Dominguez-Pinilla N, Allende $L M$, et al. No overt clinical immunodeficiency despite immune biological abnormalities in Patients with constitutional mismatch repair deficiency. Front Immunol. 2018;9:1506.

15. Win AK, Jenkins MA, Buchanan DD, Clendenning M, Young JP, Gliles GG, et al. Determining the frequency of de novo germline mutations in DNA mismatch repair genes. J Med Genet. 2011;48:530-4.

16. Wimmer K, Rosenbaum T, Messiaen L. Connections between constitutional mismatch repair deficiency syndpome and neurofibromatosis type 1. Clin Genet. 2017:91:507-19.
17. Wang Q, Montmain G, Ruano E, Upadhyaya M, Dudley S, Liskay RM, et al. Neurofibromatosis type 1 gene as a mutational target in a mismatch repair-deficient cell type. Hum Genet. 2003;112:117-23.

18. Shah KN. The diagnostic and clinical significance of café-au-lait macules. Pediatr Clin N Am. 2010;57:1131-53.

19. Suerink M, Ripperger T, Messiaen L, Menko FH, Bourdeaut F, Colas C, et al. Constitutional mismatch repair deficiency as a differential diagnosis of neurofibromatosis type 1: consensus guidlines for testing a child without malignancy. J Med Genet. 2018;0:1-10.

20. Ricciardone MD, Ozçelik T, Cevher B, Ozdağ H, Tuncer M, Gürgey A, et al. Human MLH1 deficiency predisposes to hematological malignancy and neurofibromatosis type 1. Cancer Res. 1999;59:290-3.

21. Wan Q, Lasset C, Desseigne F, Frappaz D, Bergeron C, Navarro C, et al. Neurofibromatosis and early onset of cancers in hMLH1-deficient children. Canser Ras. 1999;59:294-7.

\section{Publisher's Note}

Springer Nature remains neutral with regard to jurisdictional claims in published maps and institutional affiliations.
Ready to submit your research? Choose BMC and benefit from:

- fast, convenient online submission

- thorough peer review by experienced researchers in your field

- rapid publication on acceptance

- support for research data, including large and complex data types

- gold Open Access which fosters wider collaboration and increased citations

- maximum visibility for your research: over $100 \mathrm{M}$ website views per year

At BMC, research is always in progress.

Learn more biomedcentral.com/submissions 